\title{
Investigation of reproducibility of differentially expressed genes in DNA microarrays through statistical simulation Xiaohui Fan ${ }^{1}$, , Leming Shi ${ }^{1}$, Hong Fang ${ }^{3}$, Stephen Harris ${ }^{1}$, Roger Perkins ${ }^{3}$ and Weida Tong*1
}

\author{
Address: ${ }^{1}$ National Center for Toxicological Research (NCTR), US Food and Drug Administration, 3900 NCTR Rd., Jefferson, AR 72079, USA, \\ 2Pharmaceutical Informatics Institute, College of Pharmaceutical Sciences, Zhejiang University, Hangzhou 310027, PR China and ${ }^{3}$ Z-tech \\ Corporation, an ICF International, National Center for Toxicological Research (NCTR), US Food and Drug Administration, 3900 NCTR Rd., \\ Jefferson, AR 72079, USA \\ Email: Xiaohui Fan - fanxh@zju.edu.cn; Leming Shi - Leming.Shi@fda.hhs.gov; Hong Fang - Hong.Fang@fda.hhs.gov; \\ Stephen Harris - Steve.Harris@fda.hhs.gov; Roger Perkins - Roger.Perkins@fda.hhs.gov; Weida Tong* - Weida.Tong@fda.hhs.gov \\ * Corresponding author
}

from First International Conference on Toxicogenomics Integrated with Environmental Sciences (TIES-2007)

Raleigh, NC, USA. 25-26 October 2007

Published: 10 March 2009

BMC Proceedings 2009, 3(Suppl 2):S4

This article is available from: http://www.biomedcentral.com/I753-656I/3/S2/S4

(c) 2009 Fan et al; licensee BioMed Central Ltd.

This is an open access article distributed under the terms of the Creative Commons Attribution License (http://creativecommons.org/licenses/by/2.0), which permits unrestricted use, distribution, and reproduction in any medium, provided the original work is properly cited.

\begin{abstract}
Recent publications have raised concerns about the reliability of microarray technology because of the lack of reproducibility of differentially expressed genes (DEGs) from highly similar studies across laboratories and platforms. The rat toxicogenomics study of the MicroArray Quality Control (MAQC) project empirically revealed that the DEGs selected using a fold change (FC)based criterion were more reproducible than those derived solely by statistical significance such as $\mathrm{P}$-value from a simple t-tests. In this study, we generate a set of simulated microarray datasets to compare gene selection/ranking rules, including P-value, FC and their combinations, using the percentage of overlapping genes between DEGs from two similar simulated datasets as the measure of reproducibility. The results are supportive of the MAQC's conclusion on that DEG lists are more reproducible across laboratories and platforms when FC-based ranking coupled with a nonstringent $\mathrm{P}$-value cutoff is used for gene selection compared with selection based on $\mathrm{P}$-value based ranking method. We conclude that the MAQC recommendation should be considered when reproducibility is an important study objective.
\end{abstract}

\section{Background}

The utility of DNA microarrays has been demonstrated in clinical applications and risk/safety assessments [1-6]. With the wide variety of array platforms and analysis approaches, however, challenges remain in this field. For example, several publications [7-11] recently raised concerns about the reliability of microarray technology based on the lack of agreement in differentially expressed genes (DEGs) obtained from different laboratories and array platforms for highly similar study designs and experiments. By reanalyzing seven of the largest public DNA microarrays datasets aimed at cancer prognosis, Michiels et al. found that the signature genes of the classifiers were extremely unstable [11]. The MicroArray Quality Control 
(MAQC) project conducted a large study using reference RNA samples and a toxicogenomics dataset $[12,13]$ revealed that the DEGs selected using fold change (FC)based criterion were more stable in terms of reproducibility across labs and platforms than those derived solely from statistical significance measures such as P-value from simple t-tests. The MAQC study caused some to argue that the MAQC conclusion could be so broadly generalized. In response, this study sought to duplicate the finding of MAQC, except through statistical simulation using postulated datasets. Specifically, we generated a set of simulated microarray datasets with varying amount of noise, expression magnitude, and sample size in order to systematically compare the relationships among gene selection/ ranking rules (i.e., P-value, FC and their combinations) with respect to reproducibility of DEGs.

\section{Methods}

Two simulated groups of samples were generated, a control group and a treatment group. The control and treatment groups consisted of either 5 or 50 replicates (samples) with each replicate containing 12,000 genes. The gene intensities of the samples in the control group were simulated by Signal + Noise while the corresponding gene intensities of the treated samples were Signal + FC + Noise. Both Signal and Noise were distributed normally, while FC was distributed exponentially. The study used the set of parameters that are summarized in Table 1. Specifically, both treated and control groups contain either 50 or 5 simulated replicates with a distributed CV (coefficient of variation) similar to those observed in the MAQC study for the reference RNA samples and rat toxicogenomics dataset. CV values of $2 \%, 10 \%, 30 \%$, and $100 \%$ were used, corresponding to low, medium, high, and very high noise level, respectively. For each CV value, three expression magnitudes were considered corresponding to mean FC of 1.5, 0.6 and 0.2; these values are corresponding to the MAQC's study for the reference RNA samples and rat toxicogenomics dataset as well as consistent with the range typically found in clinical microarray experiments, respectively.

\section{Results and discussions}

The study applied 24 simulated conditions (or 24 permutations) corresponding to two sample sizes, each having four values of CV and three different mean FC values, corresponding to Table 1 . For each permutation, six gene selection methods were used to determine DEGs by comparing the treated group with the control group. These gene selection methods were (1) FC: genes are rank ordered by FC and DEGs determined by a FC cut-off only; (2-3) FC $(P<0.01)$ and FC $(P<0.05)$ : genes are rank ordered first by FC and DEGs are determined by a P-value cutoff of either 0.01 and 0.05 ; (4) $P$ : genes are rank ordered by P-value from the simple t-test and DEGs are selected using a specified P-value cutoff; and (5-6) P (FC $>1.4)$, and $P(F C>2)$ : genes are rank ordered first by $\mathrm{P}-$ value and DEGs are then determined by either a $\mathrm{FC}=1.4$ or FC $=2$ cutoff. Each permutation was repeated twice to mimic the process of conducting the same experiment in two different labs or two different platforms. The resulting DEGs from two simulations were compared to assess reproducibility across labs or platforms based on the percentage of overlapping genes (POG).

Figure 1 compares six gene selection methods applied to four datasets, each containing a different noise level (i.e., $\mathrm{CV}=2 \%, 10 \%, 30 \%$ and $100 \%$ ), where POG is shown as a function of the number of genes selected as differentially expressed between two simulations for the same permutation (magnitude $=1.5$ and sample size $=50$ ). In general, the FC-based gene selection methods outperformed the Pbased gene selection method in terms of DEG reproducibility measured by POG. Specifically, three FC-based gene selection methods, i.e. FC, FC $(\mathrm{P}<0.01)$, and FC $(\mathrm{P}<$ $0.05)$ consistently result in the highest POG values, regardless of $\mathrm{CV}$ value. Higher noise consistently results in lower POG (i.e., DEG reproducibility), as expected. The POG consistently decreases with increasing CV. For P value selection methods, higher FC cutoff results in higher POG. All results are consistent with MAQC observations.

Figure 2 compares six gene selection methods on three datasets, each having a different magnitude level between

Table I: Summary of the parameters used in this study.

\begin{tabular}{llll}
\hline CV & Low & Medium & High \\
\cline { 2 - 4 } & $\sim 2 \%$ & $\sim 10 \%$ & $\sim 30 \%$ \\
\hline $\begin{array}{l}\text { Magnitude } \\
\text { (FC) }\end{array}$ & MAQC main study & MAQC Rat toxicogenomics & Clinical application \\
\cline { 2 - 4 } & $\sim 1.5$ & $\sim 0.6$ & $\sim 0.2$ \\
\hline Sample Size & 5 per group & & 50 per group \\
\hline
\end{tabular}



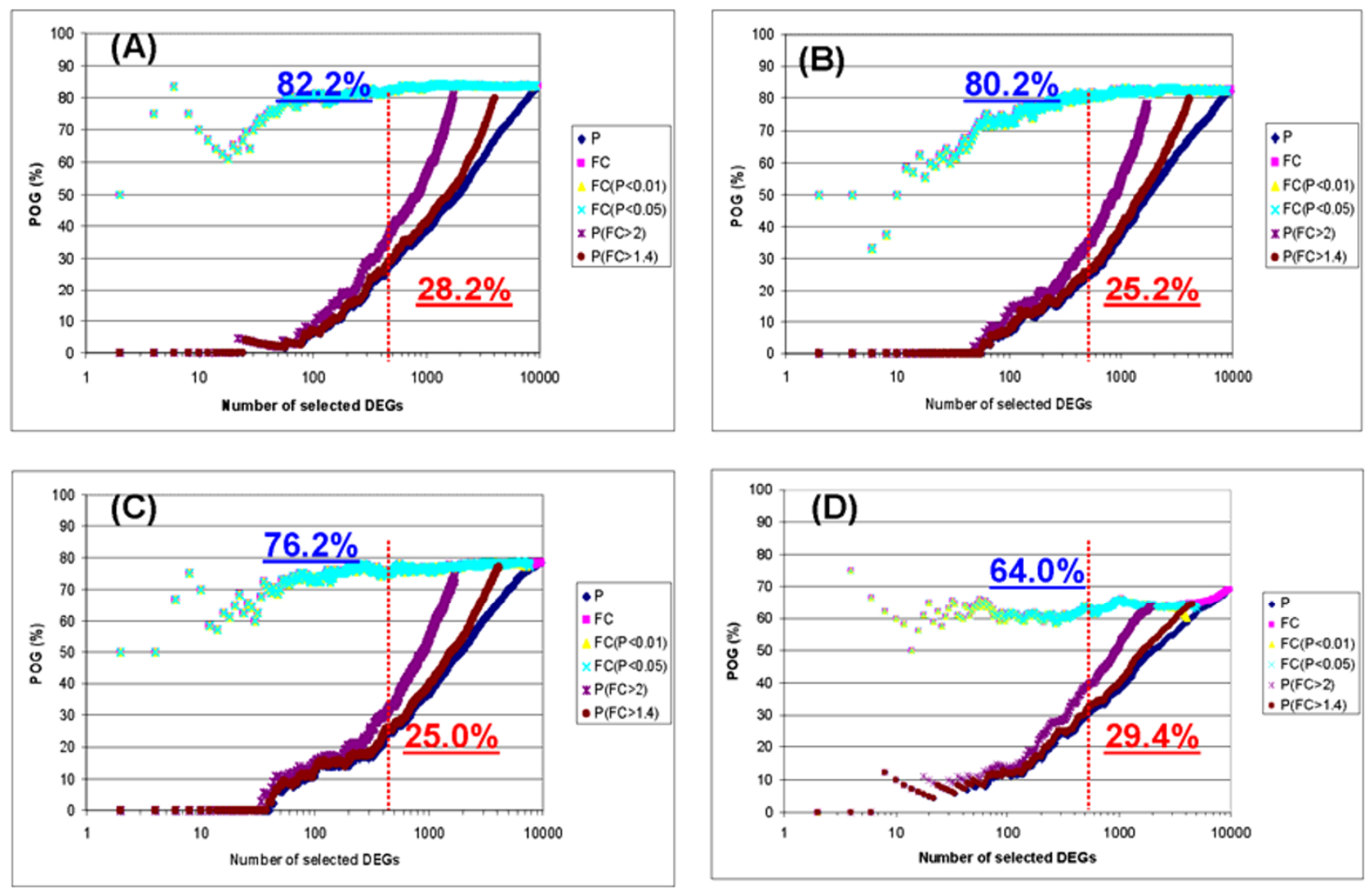

Figure I

The relationship of POGs with the degree of noise level in the simulated datasets: $(A)$ Low noise $(C V=2 \%)$; $(B)$ Medium noise ( $C V=10 \%)$; $(C)$ High noise ( $C V=30 \%)$; and $(D)$ Very high noise $(C V=100 \%)$. The simulated datasets were set to the expression magnitude difference between the treated and control groups of I.5 and the sample size of 50 . The $\mathrm{X}$-axis represents the number of genes selected as differentially expressed, and the $y$-axis represents the POG (\%) of two gene lists for a given number of differentially expressed genes. Each line on the graph represents the overlap of differentially expressed gene lists based on one of six different gene ranking/selection methods. The red and blue numbers give the POG (\%) for 500 selected DEGs (red dashed line) from $P$ rank ordering only and FC rank ordering with $P<0.05$, respectively.

the treated and control groups (i.e., $\mathrm{FC}=1.5,0.6$ and 0.2 ). Similar to Figure 1, the FC-based methods resulted in greater reproducibility compared to the P-based method. Furthermore, POG increases with increasing differential expression magnitude for FC selection methods. However, this trend is not prominent for P value-based selection methods, where it seems that the trend is equivocal.

Figure 3 compares six gene selection methods on two datasets, one having sample size of 50 and the other having sample size 5. FC-based methods again give higher POG than P value-based methods, with the larger sample size resulting in higher POG for either selection approach.

Whereas POG are affected by the degree of noise level, expression magnitude and sample size of the datasets, the above results clearly demonstrated that the DEGs become more reproducible, especially when fewer genes are selected, if the FC is included as the ranking criterion for subsequent DEGs identification. It is likely that the discordance of reported microarray results in literature is in large part due to the widespread of using P-based approach to rank genes over the FC-based method. The results of our another related study demonstrated that the relationship of the tradeoff between reproducibility and specificity/sensitivity in the FC $(\mathrm{P})$ approach can be balanced by weighting the $\mathrm{FC}$ as a primary consideration in gene ranking: that is an FC criterion explicitly incorporates the measured quantity to ensure reproducibility, whereas a P criterion incorporates control of sensitivity and specificity [14]. 


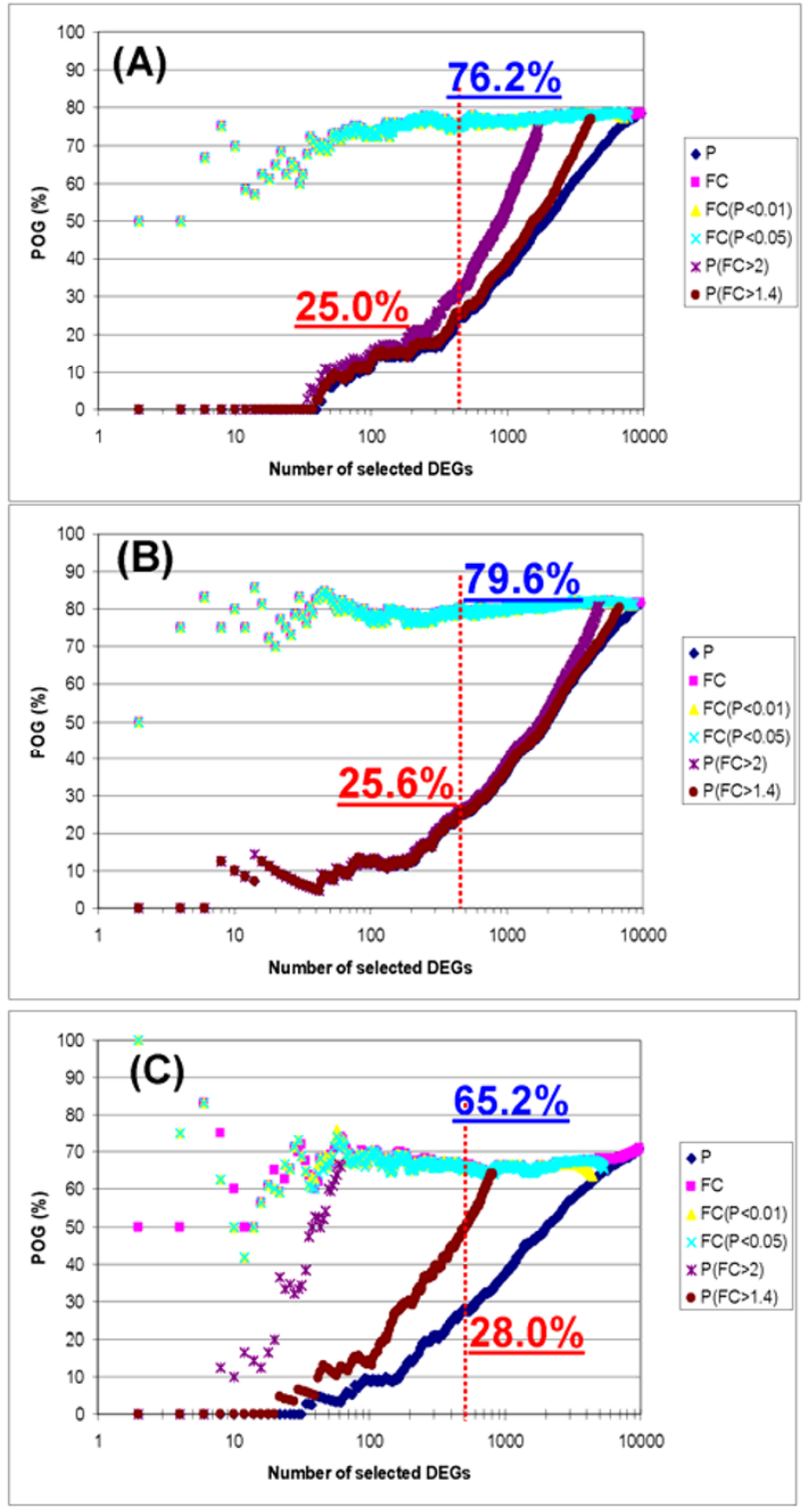

\section{Figure 2}

The relationship of POG with the degree of difference in expression magnitude between the treated versus control groups. (A) Magnitude $=0.6$; (B) Magnitude $=1.5$; and $(\mathrm{C})$ Magnitude $=0.2$. The simulated datasets had $C V=30 \%$ and sample size $=50$. The $x$-axis represents the number of genes selected as differentially expressed, and the $y$-axis represents the POG (\%) of two gene lists for a given number of differentially expressed genes. Each line on the graph represents the overlap of differentially expressed gene lists based on one of six different gene ranking/selection methods. The red and blue numbers give the POG (\%) when 500 genes (red dashed line) are selected as DEGs using $P$ rank ordering only and $\mathrm{FC}$ rank ordering with $\mathrm{P}<0.05$, respectively.

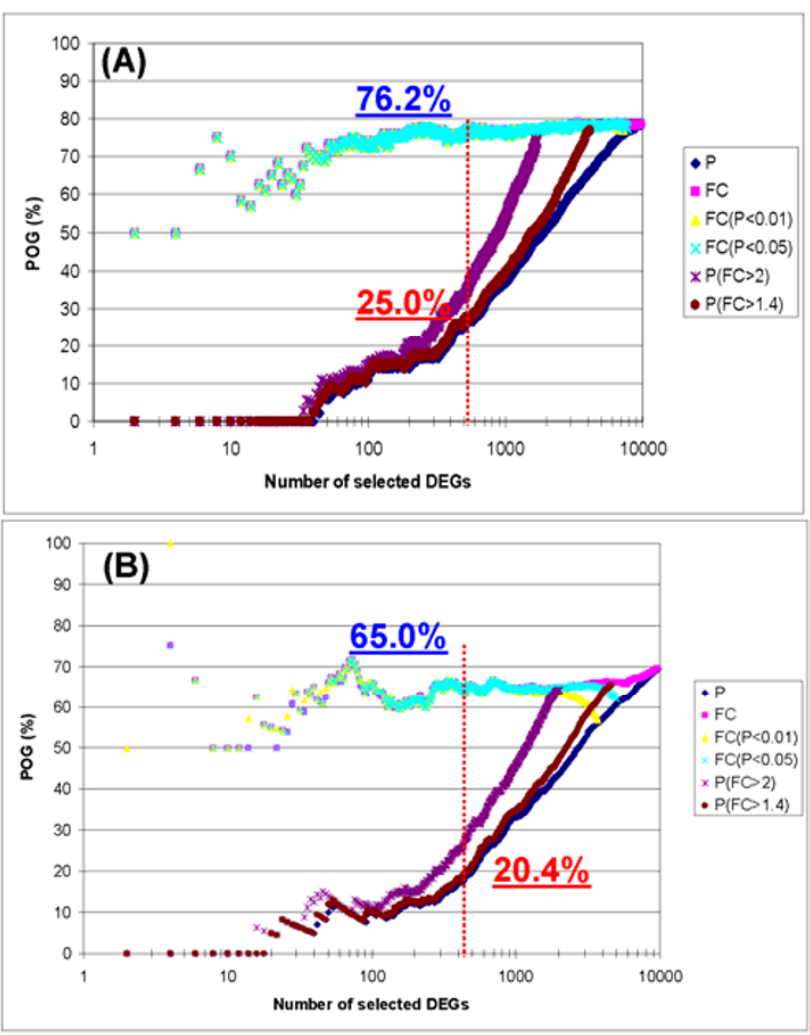

Figure 3

The relationship of POG with the sample size: (A) 50 samples/group and (B) 5 samples/group. The simulated datasets had CV $=30 \%$ and magnitude $=50$ (see Table I).

The $\mathrm{x}$-axis represents the number of genes selected as differentially expressed, and the $y$-axis represents the POG (\%) of two gene lists for a given number of differentially expressed genes. Each line on the graph represents the overlap of differentially expressed gene lists based on one of six different gene ranking/selection methods. The red and blue numbers give the POG (\%) when 500 genes (red dashed line) are selected as DEGs using $P$ rank ordering only and FC rank ordering with $P<0.05$, respectively.

\section{Conclusion}

Our simulation results show that the choice of gene selection method significantly affects apparent reproducibility of DEGs as measured by POG. Reproducibility as measured by POG between lists substantially increases when FC is the ranking criterion for identifying DEGs, especially for shorter gene lists. This observation holds for different noise levels, expression magnitudes and sample sizes. Our simulation are consistent with MAQC's conclusion that to generate more reproducible DEG lists across labs and platforms, the FC ranking with a nonstringent P-value cutoff, so named the FC (P) approach, should be considered when reproducibility is a consideration in a microarray study. 


\section{Competing interests}

The authors declare that they have no competing interests.

\section{Authors' contributions}

XF performed data analyses and finished the first draft of the manuscript. WT and HF guided the analysis and helped writing manuscript. LS had the original idea for statistical simulation. RP also helped manuscript writing. $\mathrm{SH}$ helped the microarray data management. All authors participated in preparation of the manuscript, and approved its final version.

\section{Acknowledgements}

Xiaohui Fan thanks the support by ORISE research fellowship.

The views presented in this article do not necessarily reflect those of the U.S. Food and Drug Administration

This article has been published as part of BMC Proceedings Volume 3 Supplement 2, 2009: Proceedings of the First International Conference on Toxicogenomics Integrated with Environmental Sciences (TIES-2007). The full contents of the supplement are available online at http://www.biomedcen tral.com $/ 1753-6561 / 3$ ? issue $=$ S2

\section{References}

I. Rosenwald A, Wright G, Chan WC, Connors JM, Campo E, Fisher RI, Gascoyne RD, Muller-Hermelink HK, Smeland EB, Staudt LM: The use of molecular profiling to predict survival after chemotherapy for diffuse large-B-cell lymphoma. N Engl J Med 2002, 346(25): 1937-1947.

2. van't Veer LJ, Dai HY, Vijver MJ van de, He YDD, Hart AAM, Mao M, Peterse HL, Kooy K van der, Marton MJ, Witteveen AT, et al.: Gene expression profiling predicts clinical outcome of breast cancer. Nature 2002, $415(687$ I):530-536.

3. Bhattacharjee A, Richards WG, Staunton J, Li C, Monti S, Vasa P, Ladd C, Beheshti J, Bueno R, Gillette M, et al.: Classification of human lung carcinomas by mRNA expression profiling reveals distinct adenocarcinoma subclasses. Proc Natl Acad Sci USA 200I, 98(24): I 3790- I3795.

4. Pomeroy SL, Tamayo P, Gaasenbeek M, Sturla LM, Angelo M, McLaughlin ME, Kim JYH, Goumnerova LC, Black PM, Lau C, et al.: Prediction of central nervous system embryonal tumour outcome based on gene expression. Nature 2002, 4I5(6870):436-442.

5. lizuka N, Oka M, Yamada-Okabe H, Nishida M, Maeda Y, Mori N, Takao T, Tamesa T, Tangoku A, Tabuchi $\mathrm{H}$, et al.: Oligonucleotide microarray for prediction of early intrahepatic recurrence of hepatocellular carcinoma after curative resection. Lancet 2003, 36 I (936 I):923-929.

6. Fan C, Oh DS, Wessels L, Weigelt B, Nuyten DSA, Nobel AB, van't Veer LJ, Perou CM: Concordance among gene-expressionbased predictors for breast cancer. N Engl J Med 2006, 355(6):560-569.

7. Marshall E: Getting the noise out of gene arrays. Science 2004, 306(5696):630-63i.

8. loannidis JPA: Why most published research findings are false. PLos Med 2005, 2(8):el 24.

9. Simon R: Development and evaluation of therapeutically relevant predictive classifiers using gene expression profiling. J Natl Cancer Inst 2006, 98( I 7): I I69- I I7I.

10. Ein-Dor L, Zuk O, Domany E: Thousands of samples are needed to generate a robust gene list for predicting outcome in cancer. Proc Natl Acad Sci USA 2006, I 03(I 5):5923-5928.

II. Michiels S, Koscielny S, Hill C: Prediction of cancer outcome with microarrays: a multiple random validation strategy. Lancet 2005, 365(9458):488-492.

12. Guo L, Lobenhofer EK, Wang C, Shippy R, Harris SC, Zhang L, Mei N, Chen T, Herman D, Goodsaid FM, et al.: Rat toxicogenomic study reveals analytical consistency across microarray platforms. Nat Biotechnol 2006, 24(9): I I62- I I69.

13. Shi LM, Reid LH, Jones WD, Shippy R, Warrington JA, Baker SC, Collins PJ, de Longueville F, Kawasaki ES, Lee KY, et al:: The MicroArray Quality Control (MAQC) project shows inter- and intraplatform reproducibility of gene expression measurements. Nat Biotechnol 2006, 24(9): I|5|-I|6I.

14. Shi L, Jones WD, Jensen RV, Harris SC, Perkins RG, Goodsaid FM, Guo L, Croner LJ, Boysen C, Fang $\mathrm{H}$, et al.: The balance of reproducibility, sensitivity, and specificity of lists of differentially expressed genes in microarray studies. BMC Bioinformatics 2008, 9(Suppl 9):SIO.
Publish with Biomed Central and every scientist can read your work free of charge

"BioMed Central will be the most significant development for disseminating the results of biomedical research in our lifetime. "

Sir Paul Nurse, Cancer Research UK

Your research papers will be:

- available free of charge to the entire biomedical community

- peer reviewed and published immediately upon acceptance

- cited in PubMed and archived on PubMed Central

- yours - you keep the copyright 